\title{
Ocular involvement in sarcoidosis
}

\author{
Vinay Kansal BHSc, Michael Dollin MD
}

— Cite as: CMAJ 2017 April 24;189:E609. doi: 10.1503/cmaj.160569

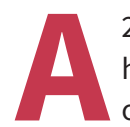

27-year-old woman presented to her family doctor with bilateral ocular pain that waxed and waned, redness and photophobia. The symptoms had progressed over several months and were followed by hoarseness and dyspnea, all of which interfered with her activities of daily living. The patient's family doctor referred her simultaneously to an otolaryngologist and a retina specialist.

The patient's visual acuity was 20/150 bilaterally. Anterior segment examination showed conjunctival nodules on the inner surface of her lower eyelids (Figure 1), keratic precipitates, inflammatory cells and flare in the anterior chamber, and posterior synechiae (Figure 2). Fundus examination showed papillitis and periphlebitis. These ocular findings were consistent with uveitis.

Laboratory workup was negative for infectious causes and showed elevated serum lysozyme, calcium and angiotensinconverting enzyme (ACE) levels. Concurrent investigation of the patient's upper airway symptoms by the otolaryngologist included biopsy of laryngeal nodules, which showed granulomatous inflammation. Multidisciplinary discussion led to a diagnosis of presumed sarcoidosis.

Sarcoidosis is a chronic, multisystem, granulomatous disease of unknown cause. ${ }^{1}$ Ocular involvement occurs in more than $75 \%$ of patients and is the presenting symptom in $20 \%-30 \%$ of cases, most commonly with uveitis and conjunctival nodules, as seen in our patient. ${ }^{2}$ Although a slit-lamp examination was required to visualize the patient's fundus findings, anterior signs of uveitis were visible without the aid of magnification. Nonspecific findings included posterior synechiae that resulted from adhesion of the inflamed iris to the lens, which caused an irregular pupil, and keratic precipitates (Figure 2) secondary to deposition of inflammatory leukocytes onto the corneal endothelium, ${ }^{2}$ the large "mutton-fat" appearance of which, in our case, suggested granulomatous disease.

The differential diagnosis of sarcoidosis is broad, including tuberculosis, Lyme disease and syphilis. Although elevated serum ACE, calcium and lysozyme levels are suggestive of the disease, definitive diagnosis is established by tissue biopsy. ${ }^{1,2}$

Goals of treatment are to restore vision and prevent complications of inflammation. Management involves local or systemic corticosteroids, and immunomodulators for severe, chronic disease. ${ }^{2}$ Visual prognosis is good in many patients but varies depending on severity and delay in presentation. Given the complex nature of ocular sarcoidosis, early referral to an ophthalmologist and multidisciplinary care are suggested. ${ }^{2}$

\section{References}

1. Sehgal VN, Riyaz N, Chatterjee K, et al. Sarcoidosis as a systemic disease. Clin Dermatol 2014;32:351-63.

2. Pasadhika S, Rosenbaum JT. Ocular sarcoidosis. Clin Chest Med 2015;36:669-83.

\section{Competing interests: None declared.}

This article has been peer reviewed.

The authors have obtained patient consent.

Affiliations: Faculty of Medicine (Kansal), University of Ottawa; University of Ottawa Eye Institute (Dollin); Ottawa Hospital Research Institute (Dollin), Ottawa, Ont.

Correspondence to: Michael Dollin, mdollin@toh.ca 